\title{
INTEGRAL CLOSURES OF NOETHERIAN INTEGRAL DOMAINS AS INTERSECTIONS
}

\author{
FREDERICK W. CALL
}

(Communicated by Wolmer V. Vasconcelos)

\begin{abstract}
Three equivalent formulations of the property that the integral closure $\bar{A}$ of a noetherian domain $A$ can be written as $\bigcap_{\bar{A}}$ at all height-one primes $p$, are given in terms of PDE, $A^{(1)}$, and bad minimal primes in completions. Examples with these properties include excellent domains and domains with a canonical module. Writing $\bar{A}$ as an intersection of DVR's is also addressed.
\end{abstract}

\section{INTRODUCTION}

If an affine domain $A$ (i.e., finitely generated as an algebra over a field) is such that $A_{p}$ is a DVR for all $p$ in the set $G$ of height-one primes of $A$, then its integral closure $\bar{A}$ can be written as $A^{(1)}:=\bigcap_{p \in G} A_{p}$. For which noetherian domains does $\bar{A}=A^{(1)}$ (in which case $A_{p}$ must be a DVR for all $p \in G)$ ? In this note we investigate a description of when $\bar{A}=\bigcap_{p \in G} \bar{A}_{p}$ in terms of PDE, $A^{(1)}$, and bad formal minimal primes, that help answer this question. Here, $\bar{A}_{p}$ means $\left(A_{p}\right)^{-}$and equals $(\bar{A})_{p}$, the localization of $\bar{A}$ at the multiplicatively closed subset $A-p$ of $A[\mathrm{~S}$, Corollary 13.27]. Examples include excellent domains and domains with (locally) a canonical module such as a homomorphic image of a Gorenstein ring.

\section{MAIN RESUlTS}

The following lemma holds for any domains $R$ and $S$, and in torsion theoretic language says simply that $Q_{Y}(S)=Q_{Y^{\prime}}(S)$.

Lemma. Let $R \subseteq S$ be domains, and let $Y \subseteq \operatorname{Spec} R$ be nonempty and generically closed (i.e., $p^{\prime} \subseteq p \in Y \Rightarrow p^{\prime} \in Y$ ). Set $Y^{\prime}=\{q \in \operatorname{Spec} S \mid q \cap R \in Y\}$ (thus, $Y^{\prime}$ is also generically closed and nonempty). Then $\bigcap_{p \in Y} S_{p}=\bigcap_{q \in Y^{\prime}} S_{q}$. Proof. It is clear the " $\subseteq$ " holds since, for each $q \in Y^{\prime}$, we have $S_{p} \subseteq S_{q}$ where $p=q \cap R$.

Conversely, if $x \in \cap S_{q}$, let $J=(S: s x)$. For every $q \in Y^{\prime}, J \nsubseteq q$ since $x \in S_{q}$. Then $I=J \cap R$ is not contained in any $p \in Y$, for if $I$ is disjoint from the multiplicatively closed subset $R-p \subseteq S$, then so is $J$, and $J$ can

Received by the editors February 8, 1993 and, in revised form, July 16, 1993.

1991 Mathematics Subject Classification. Primary 13G05, 13 B22. 
be expanded to a prime $q^{\prime}$ (not in $Y^{\prime}$ by a preceding remark) disjoint from $R-p$, i.e., $q^{\prime} \cap R \subseteq p \in Y$. But then $q^{\prime} \cap R \in Y$, so $q^{\prime} \in Y^{\prime}$, a contradiction. Therefore, for each $p \in Y$ there is an element $a \in I-p$ with $a x \in S$, proving $x \in S_{p}$.

Theorem. Let $A$ be a noetherian integral domain, $\bar{A}$ its integral closure, and set $G=\{p \in \operatorname{Spec} A \mid$ ht $p \leq 1\}$. The following conditions are equivalent:

(1) $\bar{A}=\bigcap_{p \in G} \bar{A}_{p}$.

(2) Height-one primes of $\bar{A}$ contract to height-one primes of $A$.

(3) $A \subseteq \bigcap_{p \in G} A_{p}$ is an integral extension.

(4) For all maximal ideals $m$ of $A, B:=A_{m}$, if $q \subset q^{\prime} \in \operatorname{Spec} \widehat{B}$ with $q$ minimal and $\operatorname{ht}\left(q^{\prime} \cap B\right) \geq 2$, then there is a prime strictly between $q$ and $q^{\prime}$.

Proof. (1) $\Leftrightarrow(2)$. For integral extensions, Lying-Over holds and the height of a contracted prime cannot decrease (by INC), so all the primes of $\bar{A}$ lying over a height-one prime of $A$ are height one [M, Exercise 9.8 and Theorem 9.3(i)]. Set $H_{1}=\{q \in \operatorname{Spec} \bar{A} \mid \operatorname{ht}(q) \leq 1\}$ and $H=\left\{q \in H_{1} \mid \operatorname{ht}(q \cap A) \leq 1\right\}$. Statement (2) is then $H=H_{1}$. We now use the fact that $\bar{A}$ is Krull [F, Theorem 4.3]. By the lemma, we have the equation

$$
\bar{A}=\bigcap_{q \in H_{1}}(\bar{A})_{q} \subseteq \bigcap_{q \in H}(\bar{A})_{q}=\bigcap_{p \in G}(\bar{A})_{p}=\bigcap_{p \in G} \bar{A}_{p}
$$

with the inclusion an equality if and only if $H=H_{1}$ [M, Theorem 12.3]. Thus, (1) holds if and only if $H=H_{1}$ which is (2).

$(2) \Leftrightarrow(3)$. We use the notation $Q_{U}(A):=\bigcap_{p \in U} A_{p}$ and assume without loss of generality that $A$ is local (observe: $Q_{G}(A) / A=\bigcup\left(A:_{K} I\right) / A$ the union taken over all ideals $I$ of $A$ not contained in any prime of $G$; this equality localizes at any prime $p$ since each $I$ is finitely generated). If $x \in Q_{G}(A)$, $I=\left(A:_{A} x\right)$, and $U$ is the open set $D(I) \supseteq G$, then $x \in Q_{U}(A) \subseteq Q_{G}(A)$. Thus $Q_{G}(A)=\bigcup Q_{U}(A)$, the union taken over all open $U \supseteq G$. Now [NII, Theorem 2.6.1] says $Q_{U}(A)$ is integral if and only if for each $p \notin U, Q_{Y(p)}\left(A_{p}\right)$ is integral over $A_{p}$, where $Y(p)$ is the punctured spectrum of $A_{p}$.

On the other hand, (2) is equivalent to saying if $\operatorname{dim} A_{p} \geq 2$, there are no height-one maximal ideals of $\bar{A}_{p}$.

Thus (with a change of notation), by the above two reductions, we need to show whenever $\operatorname{dim} A \geq 2$ that $Q_{Y}(A)$ is integral over $A$ where $Y$ is the punctured spectrum of $A$, if and only if there are no height-one maximals of $\bar{A}$. But this is [NI, Corollary 1.7].

(3) $\Leftrightarrow(4)$. Again, without loss of generality, $A$ is a local domain and for any open set $U$ containing $G$ we want to show that $Q_{U}(A)$ is integral over $A$ if and only if $\operatorname{dim} \widehat{A}_{q^{\prime}} / q>1$ for all primes $q \subsetneq q^{\prime}$ of $\widehat{A}$ with $q$ minimal and $q^{\prime} \cap A \notin U$. This is just [NII, Proposition 2.7.1].

Remark 1. For a simpler proof of [NII, Proposition 2.7.1] used above in the discussion of the integrality of $Q_{U}(A)$, start with Brodmann's consideration of the $A$-module finiteness of $Q_{U}(A)$ in [B, Corollary 3.7]. Then the integrality statement follows from the clever observation of Ferrand and Raynaud of reducing to the reduced case [FR, Proposition 1.1]. Also see [Mc, Chapter X].

Remark 2. PDE. Let us say for domains $A \subseteq B$ that the extension satisfies PDE if each height-one prime of $B$ contracts to a prime of height $\leq 1$ in $A$ (e.g., as 
in condition (2) of theorem). In fact, when $A$ is noetherian and condition (2) holds, every integral extension $B$ of $A$ that is also Krull satisfies PDE (as do all extensions between $B$ and $A$, e.g., as in (3) of the theorem). This follows easily from the known result that if $A \subseteq B$ is an integral extension with $A$ and $B$ both Krull, then PDE holds [F, Proposition 6.4(b)]. For example, if a domain $B \supseteq A$ is module finite (hence integral) over a noetherian domain $A$, then $B$ is noetherian and $\bar{B}$ is Krull. If the Theorem applies to $A$, then PDE holds for $A \subseteq \bar{B}$, hence for $B \subseteq \bar{B}$. This proves:

Corollary 1. If $A \subseteq B$ are domains, $A$ noetherian and $B$ module finite over $A$, and $A$ satisfies one of the equivalent conditions of the Theorem, then so does $B$.

Examples. Locally, when the noetherian domain $A$ has a canonical module, then the extension in (3) of the Theorem is module finite [A, Theorem 3.2], hence integral (cf. [B, Proposition 5.2]). Examples include complete noetherian local domains and domains finitely generated as an algebra over a field. More generally, any domain that is the homomorphic image of a Gorenstein ring has a canonical module.

If $A$ is an excellent (local) domain, then it is universally catenary, hence $\hat{A}$ is equidimensional (and catenary, of course) and thus (4) holds [M, Theorem 31.7].

In the case that one of the above examples also has the property that $A_{p}$ is a DVR for each height-one prime $p$, then $\bar{A}=\bigcap_{p \in G} A_{p}$.

For a converse we have

Corollary 2. Let $A$ be any noetherian domain and $H$ any set of height-one primes such that $A_{p}$ is a DVR for each $p \in H$. If $\bar{A}=\bigcap_{p \in H} A_{p}$, then conditions (1)-(4) hold, $A_{p}$ is a DVR for every height-one prime $p$ of $A$, and $H$ is precisely the set of all height-one primes of $A$.

Proof. Since for each $p \in H,(\bar{A})_{p}=\left(A_{p}\right)^{-}=A_{p}$, there is a unique prime $q \in \operatorname{Spec} \bar{A}$ lying over $p$, and it is of height one since $(\bar{A})_{q}=A_{p}$. Now $\bar{A}$ is Krull, so the primes $q$ so obtained must in fact be all the height-ones of $\bar{A}$. Thus condition (2) holds. LO and INC give the last statements in the corollary since height cannot decrease upon contraction.

\section{ACKNOWLEDGMENT}

The author was a visitor at Michigan State University (where the work was done) and Clark University (which provided the resources needed to prepare the manuscript) and wishes to thank them for their hospitality.

\section{REFERENCES}

[A] Y. Aoyama, Some basic results on canonical modules, J. Math. Kyoto Univ. 23 (1983), 85-94.

[B] M. Brodmann, Finiteness of ideal transforms, J. Algebra 63 (1980), 162-185.

[FR] D. Ferrand and M. Raynaud, Fibres formelles d'un anneau local noethérian, Ann. Sci. École Norm. Sup. (4) 3 (1970), 295-311.

[F] R. Fossum, The divisor class group of a krull domain, Ergeb. Math. Grenzgeb. (3), vol. 74, Springer-Verlag, New York, 1983. 
[M] H. Matsumura, Commutative ring theory, Cambridge Univ. Press, Cambridge, 1986.

[Mc] S. McAdam, Asymptotic prime divisors, Lecture Notes in Math., vol. 1023, Springer-Verlag, Berlin, 1983.

[NI] J.-I. Nishimura, On ideal transforms of noetherian rings. I, J. Math. Kyoto Univ. 19 (1979), 41-46.

[NII] _ On ideal transforms of noetherian rings. II, J. Math. Kyoto Univ. 20 (1980), 149-154.

[S] R. Y. Sharp, Steps in commutative algebra, London Math. Soc. Stud. Texts, vol. 19, Cambridge Univ. Press, Cambridge, 1990.

Department of Mathematics/Computer Science, Clark University, Worcester, MasSACHUSETTS 01610-1477 